\title{
Determinants of maximal right ventricular function: Role of septal shift
}

\author{
Uwe P. Klima, MDa \\ Myung-Yong Lee, $M D^{b}$ \\ J. Luis Guerrero, BS \\ Paul J. LaRaia, MDa \\ Robert A. Levine, $\mathrm{MD}^{\mathrm{b}}$ \\ Gus J. Vlahakes, MD
}

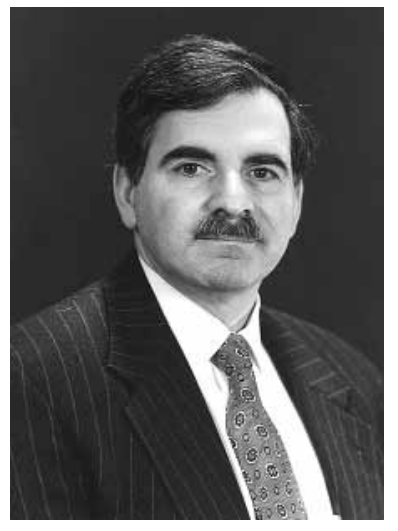

Dr Vlahakes
Background: Right heart failure can occur after orthotopic heart transplantation and can complicate implantation of left ventricular assist devices. The functional codeterminants of right ventricular function are not fully understood. We investigated the effects of left ventricular preload and afterload, systemic pressure, and the contribution of the interventricular septum to right ventricular function.

Methods and Results: In vivo studies were conducted in 12 dogs by using a highly defined, isovolumic right heart preparation. At any given arterial pressure, maximal right ventricular developed pressure was not influenced by left heart output; however, right ventricular volumes at which peak right ventricular developed pressure occurred differed significantly between the volume-loaded versus the unloaded left ventricle $(P<.05)$. A correlation was found between peak right ventricular developed pressure and mean arterial pressure. The shift of the interventricular septum toward the left ventricle is delayed under the influence of left ventricular volume load, but the maximal interventricular septal deformation does not differ at maximal right ventricular developed pressure. There was a substantial and significant decrease in peak right ventricular developed pressure when the interventricular septum was inactivated $(P<.05)$.

Conclusions: Right ventricular function has multiple determinants, including the right ventricular free wall, the left ventricle, and the interventricular septum. Changes in right ventricular performance caused by alterations in left ventricular volume load and mean arterial pressure are mediated partially through the interventricular septum, as well as through perfusion of the right ventricular free wall; inactivation of the interventricular septum leads to a significant decrease in right ventricular function. Maintaining left ventricular developed pressure and hence the contribution of the interventricular septum to right ventricular function may be important in the management of right ventricular failure.

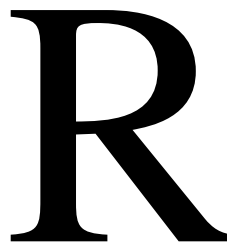

ight ventricular (RV) failure may occur in several settings: congenital heart disease, acute cor pulmonale, and acute pressure overload after heart transplantation, and is a potential source of morbidity and mortality in patients placed on left heart assistance. ${ }^{1}$ Early studies of RV function suggested that there is a relationship between systemic hemodynamics and right heart performance. ${ }^{2}$

As an extension of these early studies, Vlahakes and colleagues ${ }^{3}$ showed that right heart performance is directly related to systemic pressure and that the mechanism may involve the perfusion of the RV free wall. With increasing RV afterload, failure occurs and is associated with ischemia of the RV free wall. Increased RV afterload and preload can further complicate matters by impeding the circulation to 
the RV free-wall myocardium. ${ }^{4}$ By increasing systemic pressure, RV ischemia and failure can be reversed, suggesting that ischemia may be the mechanism of failure in RV pressure overload.

Subsequent studies have suggested that the relationship between left and right heart hemodynamics is much more complex. Page and colleagues ${ }^{5}$ demonstrated a relationship between LV and RV function. However, they showed that this effect is enhanced by the pericardium and may be independent of RV perfusion pressure, thus raising the possibility that left ventricle-right ventricle interaction may be a significant determinant of maximal RV function. Thus the right and left ventricles cannot be considered independently of one another, and the term ventricular interdependence has evolved to describe any change in the function of one ventricle that is associated with a change in the other ventricle. ${ }^{6,7}$

The purpose of this experimental study was to investigate the relationship between left ventricular (LV) preload and developed pressure and RV performance, independent of pericardial and pulmonary circulation influences. Furthermore, the contribution of interventricular septal function to right heart performance was examined. An experimental preparation was implemented that used an isovolumic right heart combined with a highly defined working left heart preparation to achieve these goals.

Additional echocardiographic studies were conducted to explore possible mechanisms responsible for the effect of left heart function on RV performance. These studies examined the relationship between interventricular septal deformity and maximal right heart function.

\section{Methods}

\section{Surgical Preparation}

The following protocol was reviewed and approved by the Subcommittee on Research Animal Care, Massachusetts General Hospital. It complies with the "Principles of Laboratory Animal Care" formulated by the National Society for Medical Research and the "Guide for the Care and Use of Laboratory Animals" prepared by the National Academy of Sciences and published by the National Institutes of Health (DHEW Publication No. 86-23, revised in 1985)

Twelve mongrel dogs (mean \pm SD weight, $24.8 \pm 3.3 \mathrm{~kg}$ ) were anesthetized with pentobarbital $(30-50 \mathrm{mg} / \mathrm{kg}$ administered intravenously) and were intubated and ventilated; anesthesia was maintained with pentobarbital infusion $\left(10 \mathrm{mg} \cdot \mathrm{kg}^{-1} \cdot \mathrm{h}^{-1}\right)$. The chest was entered through a right lateral thoracotomy in the sixth intercostal space, and the pericardium was incised and suspended to form a pericardial cradle to support the heart. The sinoatrial node was crushed to maintain a constant heart rate, and the right atrium was paced at 110 beats/min. An isovolumic model was used in which RV volume was regulated with an intracavitary balloon to precisely control RV volume (Figure 1). In this model the right ventricle was isolated from the circulation by draining systemic venous return and coronary sinus effluent to a pump oxygenator. Oxygenated blood was returned to the systemic arterial circulation

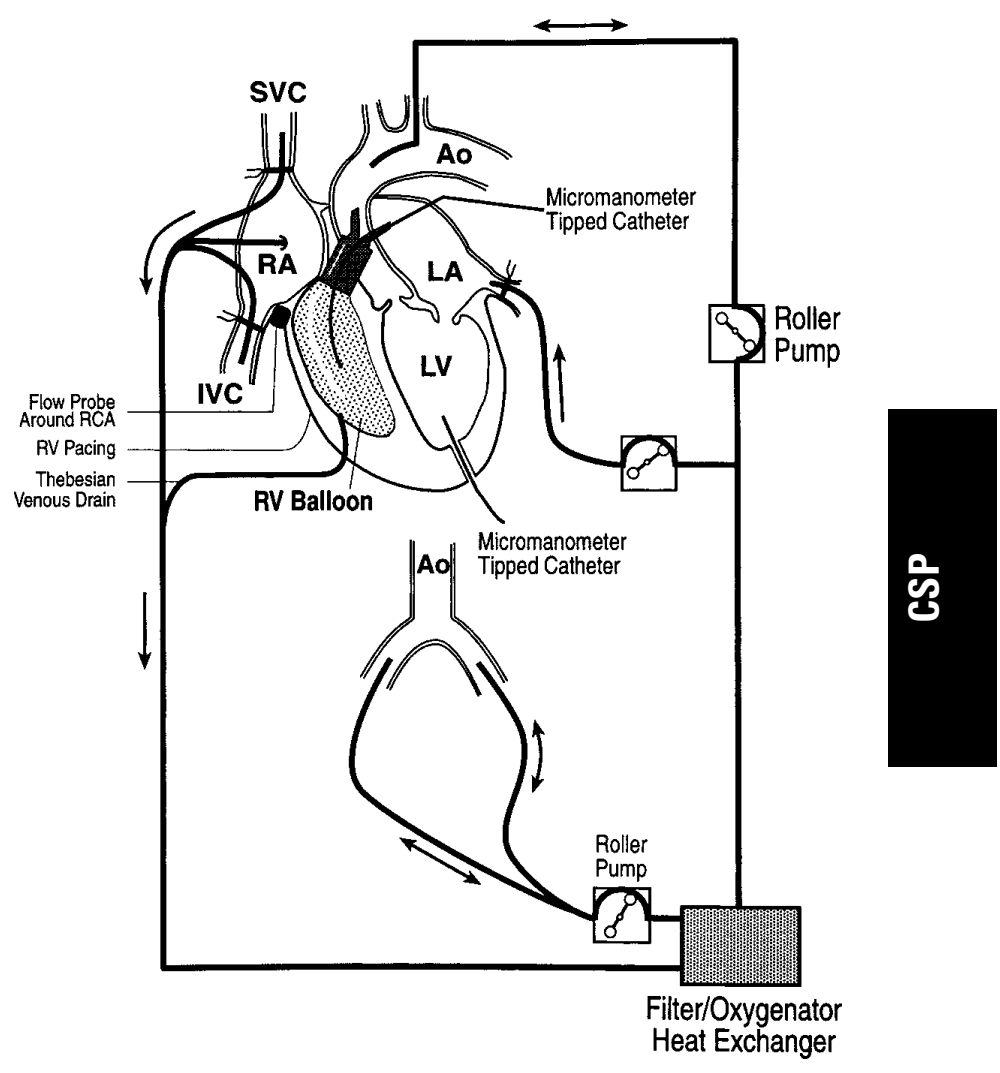

Figure 1. Schematic diagram of the experimental model. The systemic circulation is placed on cardiopulmonary bypass. LV output is determined by the inflow pumped into the left atrium, and systemic pressure (and perfusion) can be altered by pumping into or out of the arterial circulation. The right ventricle is isolated with an intracavitary balloon connected to an extracardiac tube. Through the tubing, the balloon is filled incrementally with saline solution. $R A$, Right atrium; $S V C$, superior vena cava; IVC, inferior vena cava; $L A$, left atrium; $L V$, left ventricle; $A o$, aorta.

by pumping into the left atrium, into or out of the femoral arteries, or both with separate calibrated roller pumps to control preload and afterload, respectively. The coronary arteries remained perfused directly from the ascending aorta. A high-compliance latex balloon was inserted into the right ventricle through the transected pulmonary artery. Through the right atrium, the tricuspid valve was sutured closed to prevent balloon herniation and thus to provide an absolute isovolumic model; the tricuspid valve chordae tendineae were cut to ensure that the balloon could fill the entire RV cavity and conform maximally to its cavitary contours, and Thebesian venous blood was drained with a 14-gauge cannula inserted into the $\mathrm{RV}$ apex. Conformity of the balloon shape to the RV cavity and the effectiveness of RV drainage were confirmed by echocardiographic imaging, which indicated absence of a separate space between the balloon and the RV cavity. The balloon was ligated at the pulmonary valve level around 3/4-inch diameter polyurethane tubing. During the experimental protocols, known amounts of saline solution were introduced in 10-mL increments into the RV balloon-tub- 


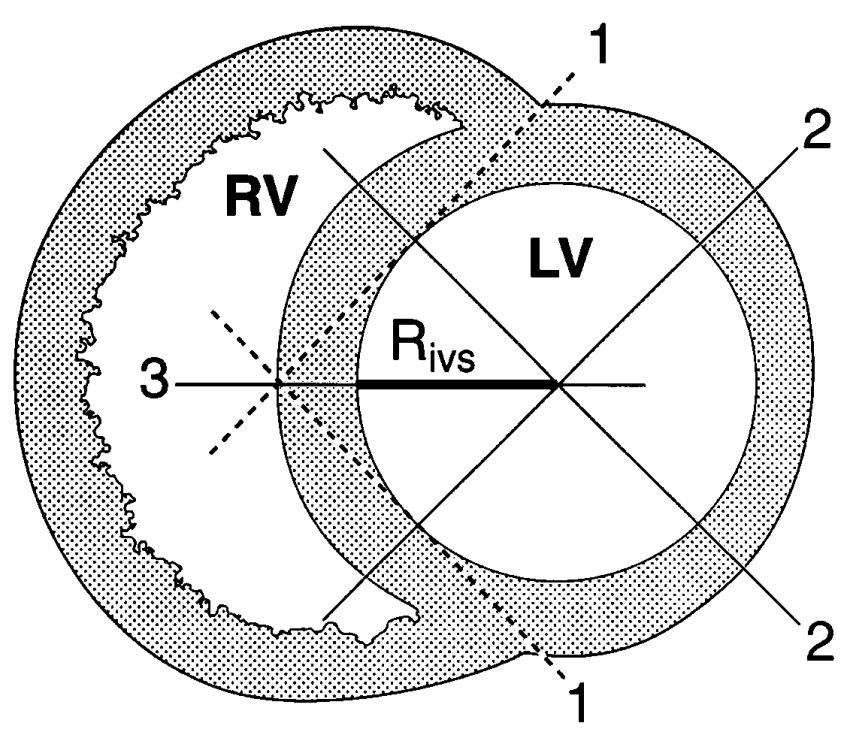

Figure 2. Measurement of $R_{\mathrm{ivs}} / \mathrm{R}_{\mathrm{i}}$ index: reference lines 1,2 lines connecting the endocardial border of the IVS; reference lines 2, 2 perpendicular lines to each of lines 1 in the middle between endocardial borders; reference line 3, measured radius of the IVS $\left(R_{i v s}\right)$. See text for further explanation.

ing system. Through a separate port at the end of the column, a micromanometer-tipped catheter (Millar Instruments, Inc, Houston, Tex) was introduced into the middle of the latex balloon. The RV balloon volume was determined as the total volume in the balloon-tubing system minus the volume in the tubing. RV developed pressure (RVDP) was calculated as RV peak systolic pressure minus RV end-diastolic pressure.

Left heart cardiac output was controlled by pumping oxygenated blood into the left atrium at a known rate. $\mathrm{LV}$ pressure was measured with a micromanometer-tipped catheter, which was placed in the LV cavity. Systemic blood pressure was measured with a fluid-filled catheter in the right internal thoracic artery, which was connected to a strain gauge (Spectramed Inc, Oxnard, Calif). Systemic pressure was adjusted and controlled independent of left heart output by pumping blood either into or out of the femoral arteries. Right coronary artery (RCA) flow was measured with an ultrasonic flow probe (Transonic Systems, Inc, Ithaca, NY) placed at the origin of the right coronary artery.

Through a small right ventriculotomy made parallel to the left anterior descending artery, the interventricular septum (IVS) was rendered nonfunctional by means of electrocoagulation to investigate the contribution to RV function from the IVS. The efficiency of this procedure was verified at the end of each experiment by staining the heart with 2,3,5-triphenyl-tetrazolium chloride and measuring the zone of septal necrosis (see "Postmortem Studies" section).

\section{Control Ventriculotomy}

To gain access to the IVS for functional inactivation, a small right ventriculotomy was necessary. It may be argued that the ventriculotomy of the right ventricle per se might contribute to impaired
$\mathrm{RV}$ function and reduced RVDP. This issue was investigated in other studies $(n=6)$; peak RVDP did not differ between measurements of RVDP taken before and after the ventriculotomy. The ventriculotomy was performed over a maximum length of $1 \mathrm{~cm}$.

\section{Experimental Protocols}

Group 1: Relation between RV failure, left heart hemodynamics, and septal function. Six preparations were studied in a series of hemodynamic stages created by increasing the volume of the RV balloon. Increments of $10 \mathrm{~mL}$ of saline solution were added at each stage until RV failure occurred: the point of RV failure was defined when a decrease in RVDP occurred with the final administered RV balloon volume increment.

This protocol was conducted at 2 left heart outputs: zero and 2 $\mathrm{L} / \mathrm{min}$. The protocol at both LV outputs was repeated at mean systemic pressures of 50, 80, and $120 \mathrm{~mm} \mathrm{Hg}$ in random order.

After thermal inactivation of the IVS, as described, the protocol was repeated at zero and $2 \mathrm{~L} / \mathrm{min}$ left heart output and at a mean arterial pressure of $80 \mathrm{~mm} \mathrm{Hg}$.

Group 2: Septal deformity and $\boldsymbol{R} \boldsymbol{V}$ function. The relationship between septal deformity and RV function was evaluated in 6 additional preparations by using an echocardiographic index of septal deformity. A 3.5-MHZ transducer (Hewlett-Packard phased array sector scanner, Model 77020A) was used for echocardiographic imaging, and images were recorded for offline analysis. For the analysis of IVS deformity, the method of King et al ${ }^{8}$ was used to determine the $\mathrm{R}_{\mathrm{ivs}} / \mathrm{R}_{\mathrm{i}}$ index, which expressed the degree of septal deformity (Figure 2 ). The short-axis view at the high papillary muscle level, the apical 4-chamber view, and the apical longaxis view were recorded to this end. All the measurements were done at end-diastole, when LV volume was maximal. The radius of the septal curvature $\left(\mathrm{R}_{\mathrm{ivs}}\right)$ was measured by the distance from the septum to an intersecting point of 2 lines perpendicular to any 2 arbitrary chords of the IVS (Figure 2, line 2); this absolute radius was subject to change according to changes in the short-axis areas of both ventricles. In the same echocardiographic frame, the LV endocardial border was traced, the area was measured, and the square root of the area was divided by $\pi$ to yield an expression of the ideal radius $\left(\mathrm{R}_{\mathrm{i}}\right)$, which is a function of LV size. The actual septal radius $\left(\mathrm{R}_{\mathrm{ivs}}\right)$ was normalized to the index of ventricular size $\left(\mathrm{R}_{\mathrm{i}}\right)$, and the resulting index $\left(\mathrm{R}_{\mathrm{ivs}} / \mathrm{R}_{\mathrm{i}}\right)$ was used to express the severity of septal deformity.

The septal deformity index was measured with increasing RV balloon volumes at 0 and $2 \mathrm{~L} / \mathrm{min}$ left heart output at $80 \mathrm{~mm} \mathrm{Hg}$ mean arterial pressure and with a functioning IVS. The protocol was repeated with an inactivated IVS.

\section{Postmortem Studies}

The left coronary artery was perfused with 2,3,5-triphenyl-tetrazolium chloride (Sigma) to determine the proportion of the IVS rendered nonviable; hearts were then incubated at $37^{\circ} \mathrm{C}$ in normal saline solution for 30 minutes and were then fixed by means of immersion in phosphate-buffered formalin $(\mathrm{pH}$ 7.0). After fixation of the heart, the macroscopically undyed areas of each region were separated and weighed. The proportion of necrotic septum was calculated by means of this technique and expressed as a percentage of the total regional weight. 
TABLE 1. Morphometric analysis

\begin{tabular}{lcc}
\hline & Mean \pm SD & Range \\
\hline Total heart weight $(\mathrm{g})$ & $185 \pm 25$ & $152-212$ \\
RV free-wall weight & $53 \pm 13$ & $33-72$ \\
LV free-wall weight & $89 \pm 11$ & $71-101$ \\
Septum weight & $43 \pm 9$ & $30-53$ \\
Proportion of necrotic IVS (\%) & $86 \pm 8$ & \\
Proportion of RV free wall supplied by RCA (\%) & $81 \pm 10$ & \\
\hline $\mathrm{n}=6$. & &
\end{tabular}

TABLE 2. Peak RVDP: Effect of left heart output, mean arterial pressure, and septal function

\begin{tabular}{llc}
\hline & \multicolumn{2}{c}{ Maximal RVDP (mm Hg) } \\
\cline { 2 - 3 } & $\begin{array}{c}\text { Left heart } \\
\text { output }= \\
\text { 0 L/min }\end{array}$ & $\begin{array}{c}\text { Left heart } \\
\text { output }= \\
\text { 2 L/min }\end{array}$ \\
\hline Mean arterial pressure & & \\
$50 \mathrm{~mm} \mathrm{Hg}$ & $47.5 \pm 12.1^{*}$ & $48.5 \pm 11.7^{*}$ \\
$80 \mathrm{~mm} \mathrm{Hg}$ & $60.7 \pm 15.2^{*}$ & $62.3 \pm 14.9^{*}$ \\
$120 \mathrm{~mm} \mathrm{Hg}$ & $77.7 \pm 17.9^{*}$ & $79.3 \pm 17.1^{*}$ \\
Working septum (at $80 \mathrm{~mm} \mathrm{Hg}$ ) & $60.7 \pm 15.4$ & $62.3 \pm 16.0$ \\
Inactivated septum (at $80 \mathrm{~mm} \mathrm{Hg})$ & $39.8 \pm 11.2 \dagger$ & $40.3 \pm 11.7 \dagger$
\end{tabular}

Values are given as means \pm SD $(n=6)$.

One-way analysis of variance with repeated measures and StudentNeuman-Keuls test, mean arterial pressure of 50 versus 80 versus $120 \mathrm{~mm}$ $\mathrm{Hg}:{ }^{*} P<.05$

Paired $t$ test: working septum versus inactivated septum, $\uparrow P<.05 ; P=$ not significant, zero versus $2 \mathrm{~L} /$ min left heart output.

The artery was perfused with blue dye (Nystral, Sigma) at a mean perfusion pressure of $80 \mathrm{~mm} \mathrm{Hg}$ to determine the proportion of the RV free wall perfused by the right coronary artery. The dyed area was excised and weighed, and its weight was compared with that of the entire RV free wall.

\section{Data Analyses}

Data are expressed as means $\pm \mathrm{SD}$. The paired $t$ test was used to compare peak RVDP between the zero and $2 \mathrm{~L} / \mathrm{min}$ left heart output configurations at the same mean arterial pressure. This test was also used to compare the RV volumes at which peak RVDP occurred for zero and $2 \mathrm{~L} / \mathrm{min}$ left heart output. One-way analysis of variance with repeated measures and the Student-NewmanKeuls test were used to determine the influence of mean arterial pressure on maximal RVDP for zero and $2 \mathrm{~L} / \mathrm{min}$ left heart output. These tests were also used to compare hemodynamic data in preparations with a working septum versus those with an inactivated septum at zero and $2 \mathrm{~L} / \mathrm{min}$ left heart outputs. It was also used to test for differences in RCA flow in the zero versus the 2 $\mathrm{L} / \mathrm{min}$ left heart output preparations at 50, 80, and $120 \mathrm{~mm} \mathrm{Hg}$ mean arterial pressure.

The baseline values of the septal deformity index $\left(\mathrm{R}_{\mathrm{ivs}} / \mathrm{R}_{\mathrm{i}}\right)$ were different in the presence or the absence of LV output. Therefore for

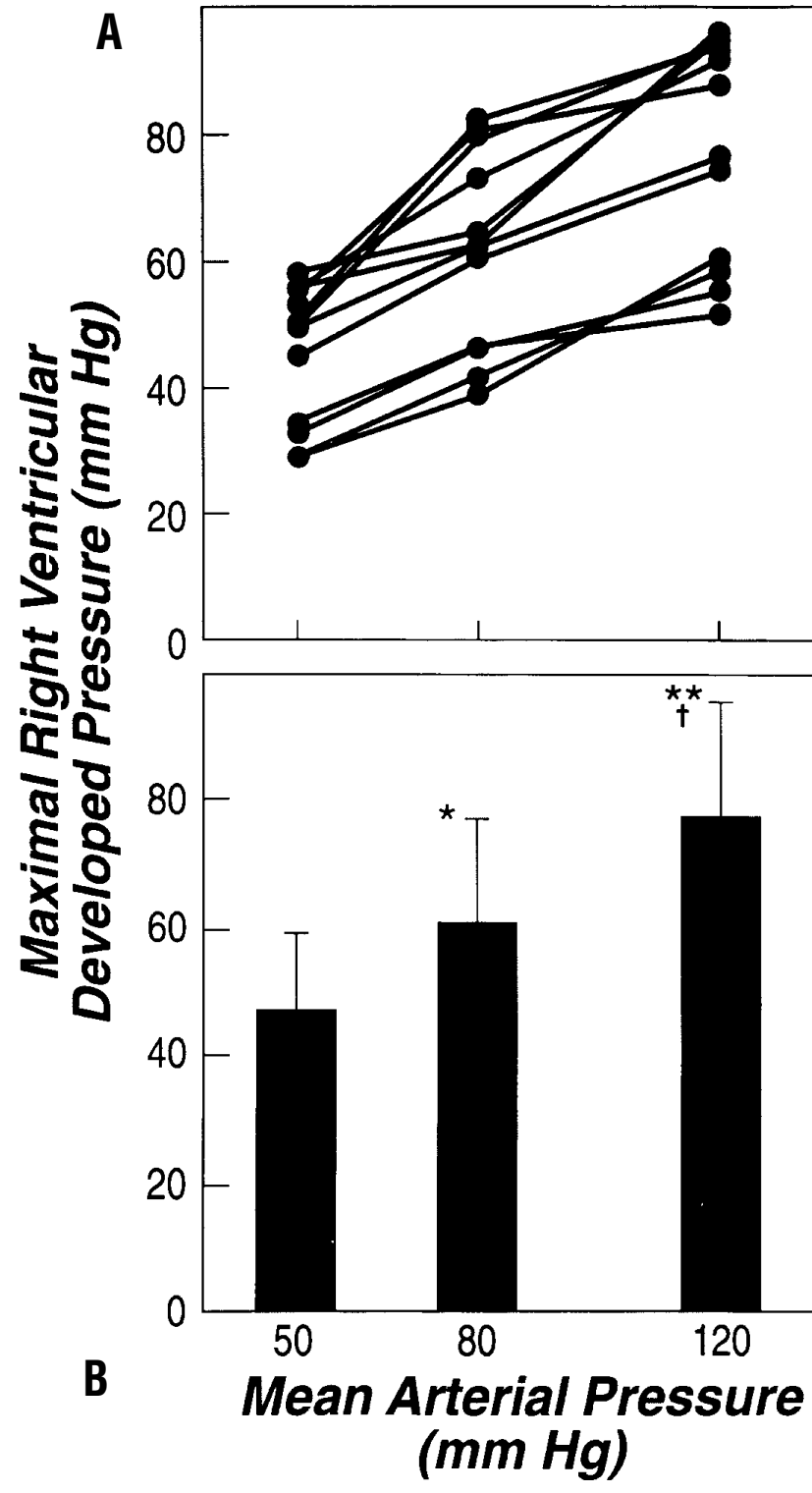

Figure 3. Influence of mean arterial pressure on maximal RVDP. Maximal RVDP values for each preparation (A) and mean values (B) are shown. For all data points shown in A, maximal RVDP equals $(0.45 \times$ mean arterial pressure $)+19.4(r=0.66 ; n=12$ [pooled data for $L V$ output $=$ zero and $L V$ output $=2 \mathrm{~L} / \mathrm{min}$ ]. ${ }^{*} P<.05$; ${ }^{* *} P<.001,1$-way analysis of variance with repeated measures and Student-Newman-Keuls test versus 50 mm Hg.

the statistical analysis of septal deformity, changes in $\mathrm{R}_{\mathrm{ivs}} / \mathrm{R}_{\mathrm{i}}$ index were expressed as the percentage change from the value at an RV balloon volume of zero. The $\mathrm{R}_{\mathrm{ivs}} / \mathrm{R}_{\mathrm{i}}$ index at maximal RVDP was compared for zero and $2 \mathrm{~L} / \mathrm{min}$ left heart output by using the paired $t$ test. This test was also used to compare the deformity index between a working and an inactivated septum.

Multiple regression analysis was used to determine factors that correlate with maximal RV function. Maximal RVDP was used as 


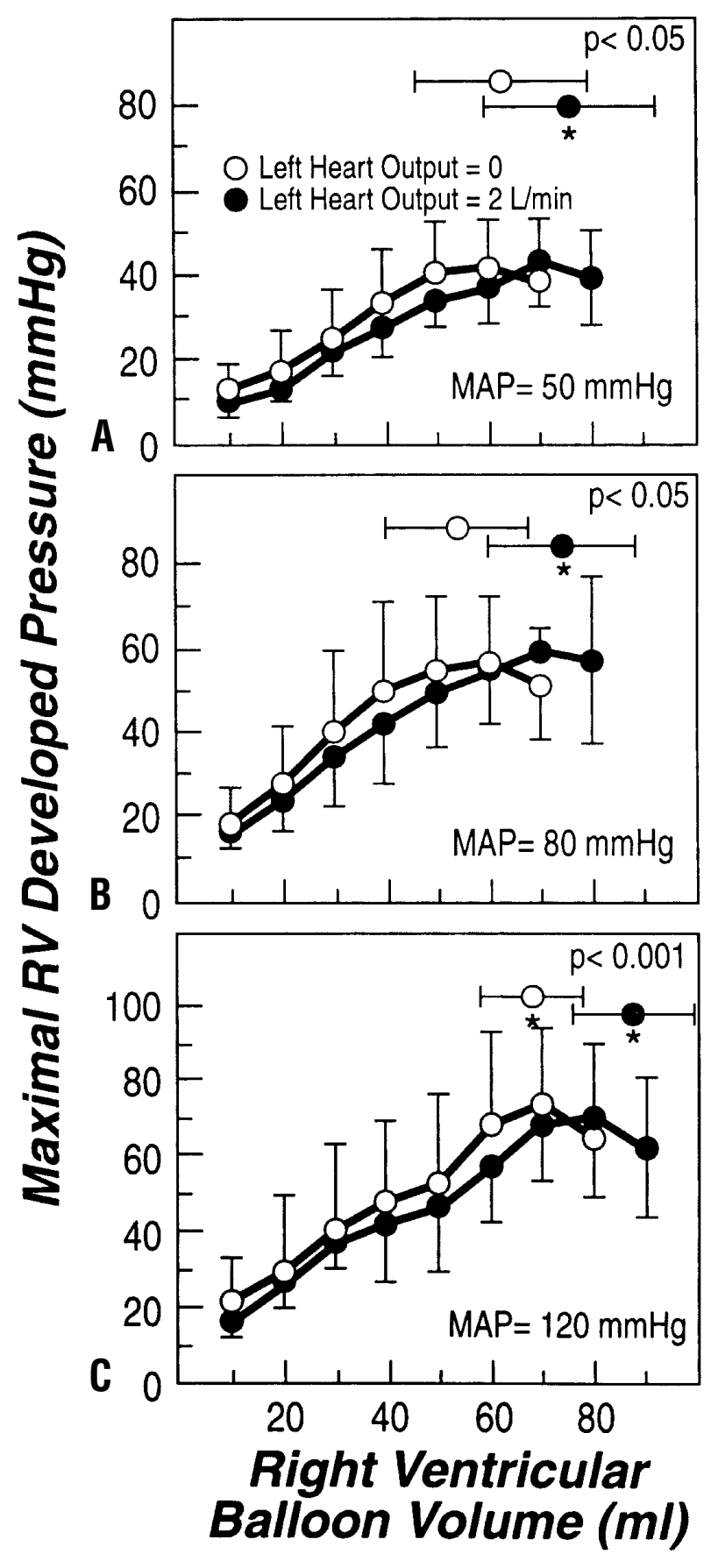

Figure 4. Influence of altered left heart output on RVDP. Symbols represent RVDP for each balloon volume at $\operatorname{dog}(n=6)$ mean arterial pressures (MAP) of 50 (A), 80 (B), and $120 \mathrm{~mm} \mathrm{Hg}$ (C). Data are expressed as means \pm SD. Increasing LV output from zero to 2 $\mathrm{L} / \mathrm{min}$ shifts the curve toward the right in a parallel manner for each balloon volume. Maximal RVDP does not differ in its peak value, but the peak occurs at different balloon volumes for zero and $2 \mathrm{~L} / \mathrm{min}$ left heart outputs. Mean RV balloon volumes that produce maximal RVDP are shown above each plot with horizontal SD bars. ${ }^{*} P<.05$, paired $t$ test, zero versus $2 \mathrm{~L} / \mathrm{min} \mathrm{LV}$ output. an independent factor, and $\mathrm{R}_{\mathrm{ivs}} / \mathrm{R}_{\mathrm{i}}$ index, $\mathrm{RV}$ volume, $\mathrm{LV}$ cardiac output, $\mathrm{LV} \mathrm{dP} / \mathrm{dt}, \mathrm{RV} \mathrm{dP} / \mathrm{dt}$, aortic pressure, left atrial pressure, systolic LV pressure, diastolic LV pressure, and RCA flow were used as dependent factors.

\section{Results}

Results of dog heart morphometric analyses are summarized in Table 1. Most of the septum was necrotic after inactivation. The majority of the RV free wall was perfused by the right coronary artery.

As shown in Table 2, maximal RVDP was not influenced by the volume of blood pumped by the left ventricle at constant systemic pressure. There was a substantial and significant difference in maximal RVDP between hearts with a working versus those with an inactivated septum. Also, as shown in Table 2 and illustrated in Figure 3, there was a statistically significant effect of mean arterial pressure (and hence developed LV pressure) on maximal RVDP.

Because at any given arterial pressure maximal RVDP was not influenced by left heart output, RVDP data at left heart outputs of zero and $2 \mathrm{~L} / \mathrm{min}$ were pooled for further analysis of maximal RVDP. The relationship between systemic arterial pressure, and hence LVDP, and maximal RVDP were examined. As shown in Figure 3, maximal RVDP increased as a function of mean arterial pressure in all preparations.

RVDP was measured at increasing balloon volumes from $10 \mathrm{~mL}$ until right heart failure occurred under conditions of zero and $2 \mathrm{~L} / \mathrm{min} \mathrm{LV}$ outputs. As shown in Figure 4, the magnitude of maximal RVDP was not influenced significantly by left heart output. However, there was a significant shift of the relationship between RV volume and RVDP to the right when left heart output was $2 \mathrm{~L} / \mathrm{min}$, with the same maximal RVDP occurring at higher balloon volumes. Figure $4, A$, represents data taken at a mean arterial pressure of $50 \mathrm{~mm} \mathrm{Hg}$, in which maximal RVDP pressure occurred at RV balloon volumes of $62 \pm 15 \mathrm{~mL}$ versus $75 \pm 19 \mathrm{~mL}$ for left heart outputs of zero and $2 \mathrm{~L} / \mathrm{min}$, respectively $(P<$ .05 ). At $80 \mathrm{~mm} \mathrm{Hg}$ mean arterial pressure (Figure 4, B), maximal RVDP occurred at RV balloon volumes of $57 \pm 14$ $\mathrm{mL}$ versus $72 \pm 15 \mathrm{~mL}$ for the 2 groups, respectively $(P<$ .05 ). At $120 \mathrm{~mm} \mathrm{Hg}$ mean arterial pressure (Figure 4, $C$ ), maximal RVDP occurred at volumes of $69 \pm 12 \mathrm{~mL}$ versus $86 \pm 15 \mathrm{~mL}$ for the 2 groups, respectively $(P<.001)$.

As shown in Table 3, RCA flow was not influenced by left heart output. Despite increasing RV balloon volume and RVDP, there was no significant increase in RCA flow in this isovolumic model; however, at the onset of RV failure, there was a significant decrease of RCA flow $(P<.05)$. RCA flow increased when systemic pressure was raised from 50 to $120 \mathrm{~mm} \mathrm{Hg}(P<.05)$.

Figure 5 illustrates that $\mathrm{R}_{\mathrm{ivs}} / \mathrm{R}_{\mathrm{i}}$ index reveals an increase of the deformity of the IVS with increasing RV volume. In 
TABLE 3. Right coronary artery flow: Effect of left heart output and mean arterial pressure

\begin{tabular}{|c|c|c|c|c|c|c|}
\hline & \multicolumn{3}{|c|}{ Zero left heart output } & \multicolumn{3}{|c|}{$2 \mathrm{~L} / \mathrm{min}$ left heart output } \\
\hline & $\begin{array}{l}\text { Low preload } \\
\text { (mL/min) }\end{array}$ & $\begin{array}{c}\text { Maximal RVDP } \\
(\mathrm{mL} / \mathrm{min})\end{array}$ & $\begin{array}{l}\text { RV failure } \\
\text { (mL/min) }\end{array}$ & $\begin{array}{l}\text { Low preload } \\
\text { (mL/min) }\end{array}$ & $\begin{array}{c}\text { Maximal RVDP } \\
(\mathrm{mL} / \mathrm{min})\end{array}$ & $\begin{array}{l}\text { RV failure } \\
\text { (mL/min) }\end{array}$ \\
\hline \multicolumn{7}{|c|}{ Mean arterial pressure } \\
\hline 50 mm Hg & $29.0 \pm 18.9^{*}$ & $30.7 \pm 18.01^{*}$ & $4.1 \pm 4.1^{*} \dagger$ & $29.3 \pm 10.8^{*}$ & $33.1 \pm 11.0^{*}$ & $1.9 \pm 1.0^{*} \dagger$ \\
\hline $80 \mathrm{~mm} \mathrm{Hg}$ & $43.2 \pm 16.1^{*}$ & $44.3 \pm 23.3^{*}$ & $13.1 \pm 10.3^{*} \dagger$ & $45.2 \pm 20.6^{*}$ & $46.1 \pm 20.4^{*}$ & $13.6 \pm 10.2^{*} \dagger$ \\
\hline $120 \mathrm{~mm} \mathrm{Hg}$ & $75.1 \pm 44^{*}$ & $76.8 \pm 44.3^{*}$ & $22.6 \pm 20.3^{*} \dagger$ & $76.5 \pm 34.2^{*}$ & $79.6 \pm 35.3^{*}$ & $18.7 \pm 8.0^{*} \dagger$ \\
\hline
\end{tabular}

Values are given as means \pm SD $(n=6)$.

One-way analysis of variance with repeated measures and Student-Neuman-Keuls test: zero versus $2 \mathrm{~L} / \mathrm{min}$ left heart output: $P=$ not significant under all conditions of RV balloon volume and mean arterial pressure; RCA flow at 50 versus 80 versus $120 \mathrm{~mm} \mathrm{Hg}$ mean arterial pressure, ${ }^{*} P<.05$; RCA flow at low preload versus maximum flow versus flow at onset of RV failure, $\uparrow P<.05$.

TABLE 4. Maximal RVDP: Effect of septal function and septal shift in working and inactivated septum

\begin{tabular}{|c|c|c|c|c|}
\hline & \multicolumn{4}{|c|}{ Mean arterial pressure $=\mathbf{8 0} \mathbf{m m ~} \mathbf{~ g g}$} \\
\hline & \multicolumn{2}{|c|}{ Working septum } & \multicolumn{2}{|c|}{ Inactivated septum } \\
\hline & Zero left heart output & $2 \mathrm{~L} / \mathrm{min}$ left heart output & Zero left heart output & $2 \mathrm{~L} / \mathrm{min}$ left heart output \\
\hline Maximal RVDP (mm Hg) & $60.4 \pm 14.4$ & $62.1 \pm 14.3$ & $39.8 \pm 16.5^{*}$ & $38.5 \pm 8.8^{*}$ \\
\hline Maximal positive RV dP/dt & $1220 \pm 230$ & $1380 \pm 290$ & $640 \pm 250^{*}$ & $750 \pm 210^{*}$ \\
\hline RV volume at maximal RVDP & $54 \pm 19$ & $66 \pm 18$ & $50 \pm 7$ & $62 \pm 11$ \\
\hline $\mathrm{R}_{\mathrm{ivs}} / \mathrm{R}_{\mathrm{i}}$ increase at maximal RVDP (\%) & $50.2 \pm 37.0$ & $63.1 \pm 66.2$ & $55.9 \pm 42.4$ & $73.5 \pm 58.6$ \\
\hline
\end{tabular}

Values are given as means $\pm S D(n=6)$.

Paired $t$ test: zero versus $2 \mathrm{~L} /$ min left heart output in working versus inactivated septum, ${ }^{*} P<.001$; zero versus $2 \mathrm{~L} /$ min left heart output, $P=$ not significant.

each dog studied, when the RV volumes were identical (50 $\mathrm{mL}$ ), the percentage changes of deformity indices were significantly different between left heart outputs of 0 and 2 $\mathrm{L} / \mathrm{min}\left(\mathrm{R}_{\mathrm{ivs}} / \mathrm{R}_{\mathrm{i}}\right.$ index, $57 \pm 40$ vs $27 \pm 28$, respectively; $P<$ $.05)$. The deformity index at maximal RVDP, however, did not differ significantly between left heart outputs of zero and $2 \mathrm{~L} / \mathrm{min}\left(\mathrm{R}_{\mathrm{ivs}} / \mathrm{R}_{\mathrm{i}}\right.$ index, $58 \pm 20$ vs $56 \pm 12$, respectively; $P=$ not significant). These data suggest that under the influence of increased left heart output, more RV preload is needed to shift the septum, but at the point of maximal RVDP, there was no greater magnitude of the shift of the IVS.

As shown in Table 4, there was no difference in $R_{i v s} / R_{i}$ index at maximal RVDP, irrespective of whether the septum was working or inactivated, even though there was a significant difference in maximal RVDP and maximal RV dP/dt.

\section{Discussion}

This study demonstrates that the IVS and left heart hemodynamics contribute significantly to RV function. A linear correlation was demonstrated for systemic arterial pressure, a reflection of peak developed LV pressure, and RV performance. This issue has been explored by previous investigators, who showed that LV unloading impairs RV function ${ }^{9}$ and that a decrement in septal function with LV unloading may be the responsible mechanism. ${ }^{10}$

It has been shown previously that when RV pressure is increased acutely, RV failure occurs and is associated with ischemia of the right ventricle. ${ }^{3,11}$ This observation led to the notion that under conditions of increasing RV afterload, maximal RV function is determined primarily by myocardial perfusion and the ischemic threshold of the RV free wall; the right coronary driving pressure, defined as the difference between mean RCA pressure and mean RV pressure $^{4,12}$ might not be sufficient to avoid RV free-wall ischemia and consequent failure as RV afterload is increased. As a corollary to this observation, these studies demonstrated that RV function could be improved and RV failure could be reversed simply by increasing systemic arterial pressure. ${ }^{2,3}$ It was hypothesized that the salutary effect on RV function was mediated by improved RV myocardial perfusion, relief of ischemia, and, consequently, improved RV free-wall function. These studies taken together would suggest that there should be a relationship between RV pressure load and RV free-wall blood flow. However, the issue of RV myocardial blood flow and RV demand has been controversial in the literature, with some 


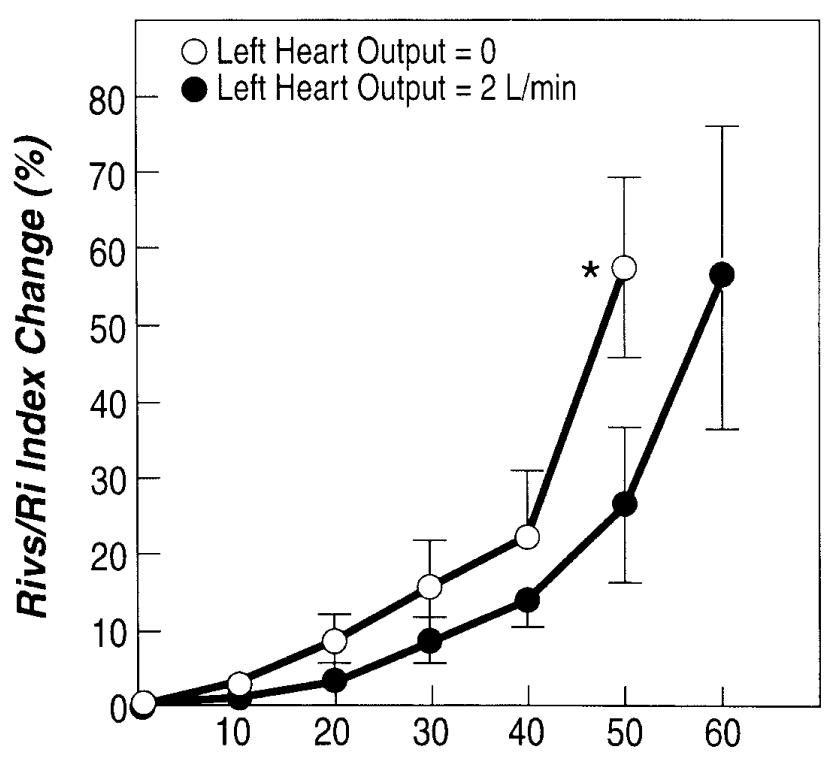

Right Ventricular Balloon Volume ( $\mathrm{ml})$

Figure $5 . R_{i v s} / R_{i}$ index reveals an increase in the deformity of the IVS with increasing RV volume ( $n=6)$. At increasing $L V$ volumes, larger RV volumes are required to produce the same degree of septal deformity.

investigators showing an increase in right coronary artery flow during RV pressure load ${ }^{12-14}$ and others showing less effect. ${ }^{15,16}$ In subsequent studies in which right coronary perfusion pressure was held constant, ${ }^{5,17}$ maximal RVDP was still influenced primarily by LV developed pressure and not by right coronary perfusion pressure. Furthermore, as shown in Table 3 , in the present study there was only a small, statistically nonsignificant increase in right coronary flow from minimum to maximum RVDP. In fact, at the onset of RV failure, there was a significantly lower right coronary flow compared with that observed at minimum RV preload and less than the observed peak right coronary flow, which is consistent with previous findings that increased RV pressure can interfere with RV perfusion. ${ }^{4}$

Thus, these findings call into question earlier fundamental hypotheses and mechanisms relating overall RV performance to RV free-wall perfusion and function. ${ }^{5}$ The strong correlation between mean arterial pressure and hence developed LV pressure and right heart performance and the variable results from studies examining the role of RV free-wall perfusion suggest that the determinants of RV function are much more complex. The relationship between left and right heart hemodynamics, independent of RV free-wall perfusion, suggests that ventricular interactions may be the most significant determinants of maximal RV performance.

Two types of ventricular interactions have been hypothesized: (1) the series effect (ie, because the ventricles are connected in a series, the output of one must influence the output of the other) and (2) direct interaction, often referred to as ventricular interdependence (ie, forces are transmitted directly form one ventricle to the other, possibly influenced by the pericardium). ${ }^{7}$ Although the relative contribution of each remains controversial, more recent studies have explored and have attempted to quantify the magnitude of direct interaction.

These studies have shown that such ventricular interaction is rapid and can occur on a beat-to-beat basis ${ }^{18}$ and that the left ventricle may contribute from one fifth to two thirds of RV function. ${ }^{19-22}$ The present study confirms that the IVS contributes an important part of ventricular interaction. In this study with an open pericardium, inactivating the IVS reduced maximal RVDP by approximately one half $(P<$ $.05)$. This finding, combined with observations in the literature, suggests that septal shortening (contractile function) and septal compliance are important factors in the coupling of LV and RV systolic functions. In the present study electrocoagulation reduces septal function and compliance, similar to the effect produced by glutaraldehyde injection, ${ }^{23}$ and decreases the LV contribution to RV function; in other experimental models, increasing compliance by volume loading (and maintaining contractile function) increased ventricular coupling. ${ }^{24}$ When the IVS was inactivated by cutting (rather than by tissue coagulation or fixation), thus increasing compliance and decreasing contractile function, there was minimal effect on RV function. ${ }^{19,23}$ This latter observation suggests that the contractile function of the septum, independent of systolic deformity, is also an important determinant of RV function.

The present study suggests that septal position may determine the magnitude of left ventricle-right ventricle interaction. The end-diastolic position of the septum influences septal motion during systole,${ }^{25-27}$ suggesting that septal position may be a form of septal preload. The findings in the present study support this hypothesis. With an isovolumic right heart preparation, maximal RVDP was not influenced by left heart output (LV volume load); however, left heart output influenced the RV preload at which maximal RVDP occurred. At higher LV volumes, greater RV preload was needed to produce septal shift (ie, septal preload), with a right shift in the RV function curves shown in Figure 4. As shown by echocardiographic analysis in this model, the IVS shifts with increases in RV preload: the $\mathrm{R}_{\mathrm{ivs}} / \mathrm{R}_{\mathrm{i}}$ index of septal curvature correlated with the incremental preload of the RV balloon (Figure 5). Increasing LV preload interacts with RV preload and delays the shift of the IVS toward the left ventricle with increasing RV preload, but the magnitude of maximal IVS deformation does not differ at maximal RVDP. $\mathrm{R}_{\mathrm{ivs}} / \mathrm{R}_{\mathrm{i}}$ index at maximal RVDP did not differ significantly with changes in LV preload, even though higher $\mathrm{RV}$ balloon volumes were observed under an LV volume 
load of $2 \mathrm{~L} / \mathrm{min}$. As shown in Figure 4, the effect of this is to shift the function curve to the right, but the magnitude of maximal RVDP does not change. This is consistent with the hypothesis that septal position reflects septal preload and that the IVS contributes a major portion of RV function (ie, once sufficient septal shift [septal preload] is achieved, the same contribution to maximal RVDP is reached).

It may also be hypothesized that under the influence of LV filling, circumferential fibers shared by both ventricles $^{6,25,26}$ may be activated and are responsible for the fact that the right ventricle can accommodate higher preload (balloon volume) at identical peak RVDP. Interestingly, even though echocardiographic data were standardized, no particular threshold for the $\mathrm{R}_{\mathrm{ivs}} / \mathrm{R}_{\mathrm{i}}$ index could be determined, at which the onset of RV failure could be predicted.

\section{Critique and Limitations of the Method}

This study was conducted in a highly defined, experimental model designed to answer specific physiology questions and to avoid potential complicating physiologic issues. An isovolumic RV model was selected. With progressive RV volume or pressure loading, tricuspid regurgitation can occur, which can substantially complicate the analysis of RV function. In the intact circulation, changes in left heart hemodynamics can affect RV afterload. Furthermore, because of the asymmetric shape of the right ventricle and the ability of the ventricle to change shape with loading, accurate estimation of RV volume can be challenging. Accordingly, an isovolumic model was chosen to obviate these concerns.

In this study septal position was used as an expression of septal preload. In the strictest sense, however, septal thickness may more directly reflect true septal preload, as described by previous investigators, ${ }^{10}$ with septal position acting as an indirect surrogate.

A small right ventriculotomy was necessary to gain access to the IVS for functional inactivation by means of electrocoagulation. It may be argued that the right ventriculotomy per se is responsible for impaired RV function and reduced RVDP. However, we investigated this issue in pilot studies; peak RVDP was not influenced by the right ventriculotomy, which was performed over a total length of approximately $1 \mathrm{~cm}$. The fact that the right ventriculotomy did not alter RV function further supports and is consistent with the hypothesis that the IVS contributes a substantial amount to global RV function.

A potential criticism of the preparation is that this complex setting did not permit investigation with a closed pericardium. As suggested by some investigators, ${ }^{5,27-31}$ a closed pericardium enhances systolic ventricular interaction; thus the maximum RVDP that can be generated is less with an open pericardium than in the native state with the pericardium closed. ${ }^{5}$ Furthermore, the displacement of the IVS might actually be less in the open pericardium versus in a closed pericardium preparation because of the ability of the RV free wall to expand outward further than a closed pericardium would permit. Thus the balloon might now expand the RV free wall more with the pericardium open, and the force developed at the IVS might be less. Caution is necessary when drawing conclusions from the open pericardium preparation and relating it to human hearts, except perhaps when analyzing the postoperative state.

\section{Implications for Clinical Management}

RV failure can complicate cardiac surgical procedures, such as cardiac transplantation or mechanical LV assistance. This experimental study emphasizes the importance of maintaining LV pressure work to maximize RV function. In the case of cardiac transplantation, maintenance of LV developed pressure is critical, and the findings support the notion that nonspecific vasodilators that affect the systemic circulation may be detrimental in this clinical setting. In the case of mechanical support of the failing left ventricle, this can cause a significant decrement in RV function, ${ }^{9}$ creating a significant challenge and underscoring the importance of having management strategies that permit the left ventricle to perform pressure work if possible.

\section{References}

1. Oakley C. Importance of right ventricular function in congestive heart failure. Am J Cardiol. 1988;62:14A-9A.

2. Salisbury PF. Coronary artery pressure and strength of right ventricular contraction. Circ Res. 1955;3:633-8.

3. Vlahakes GJ, Turley K, Hoffman JIE. The pathophysiology of failure in acute right ventricular hypertension: hemodynamic and biochemical correlations. Circulation. 1981;63:87-95.

4. Vlahakes GJ, Verrier ED, Turley K, Hoffman JIE. Maximal vascular conductance in right ventricular myocardial circulation. Am J Physiol. 1994;266:H1363-72.

5. Page RD, Harringer W, Hodakowski GT, Guerrero JL, LaRaia PJ, Austen WG, et al. Determinants of maximal right ventricular function. J Heart Lung Transplant. 1992;11:90-8.

6. Maughan WL, Sunagawa K, Sagawa K. Ventricular systolic interdependence: volume elastance model in isolated canine hearts. Am J Physiol. 1987;253:H1381-90.

7. Bove AA, Santamore WP. Ventricular interdependence. Prog Cardiovasc Dis. 1981;23:365-88.

8. King ME, Braun H, Goldblatt A, Liberthson R, Weyman AE. Interventricular septal configuration as a predictor of right ventricular systolic hypertension in children: a cross-sectional echocardiographic study. Circulation. 1983;68:68-75.

9. Moon MR, Castro LJ, DeAnda A, Tomizawa Y, Daughters GT III, Ingels NB Jr, et al. Right ventricular dynamics during left ventricular assistance in closed-chest dogs. Ann Thorac Surg. 1993;56:54-67.

10. Moon MR, Bolger AF, DeAnda A, Komeda M, Daughters GT III, Nikolic SD, et al. Septal function during left ventricular unloading. Circulation. 1997;95:1320-7.

11. Gold FL, Bache RJ. Transmural right ventricular blood flow during acute pulmonary hypertension in the dog: evidence for subendocardial ischemia during right ventricular failure despite residual vasodilator reserve. Circ Res. 1982;51:196-204.

12. Cross CE. Right ventricular pressure and coronary flow. Am J Physiol. 1962;202:12-6

13. Gregg DE, Pritchard WH, Shipley RE, Wearn JT. Augmentation of blood flow in the coronary arteries with elevation of right ventricular pressures. Am J Physiol. 1943;139:726-31.

14. Love WD, O'Meallie LP. Increase in coronary blood flow to the right 
atrium and ventricle in response to an acute increase in hemodynamic load. J Lab Clin Med. 1963;62:72-7.

15. Fixler DE, Archie JP, Ullyot DJ, Buckberg GD, Hoffman JIE. Effects of acute right ventricular systolic hypertension on regional myocardial blood flow in anesthetized dogs. Am Heart J. 1973;85:491-500.

16. Aukland K, Kiil F, Kjekshus J. Relationship between ventricular pressures and right and left myocardial blood flow. Acta Physiol Scand. 1967;70:116-26

17. Belenkie I, Horne SG, Dani R, Smith ER, Tyberg JV. Effects of aortic constriction during experimental acute right ventricular pressure loading. Further insights into diastolic and systolic ventricular interaction. Circulation. 1995;92:546-54.

18. Santamore WP, Damiano RJ Jr, Yamaguchi S, Taher M. Dynamic biventricular interaction during systole. Coron Artery Dis. 1990;1:298-306.

19. Yamaguchi S, Harasawa H, Li KS, Zhu D, Santamore WP. Comparative significance in systolic ventricular interaction. Cardiovasc Res. 1991;25:774-83.

20. Yaku H, Slinker BK, Bell SP, LeWinter MM. Effects of free wall ischemia and bundle branch block on systolic ventricular interaction in dog hearts. Am J Physiol. 1994;266:H1087-94.

21. Hoffman D, Sisto D, Frater RW, Nikolic SD. Left-to-right ventricular interaction with a noncontracting right ventricle. J Thorac Cardiovasc Surg. 1994;107:1496-502.

22. Li KS, Santamore WP. Contribution of each wall to biventricular function. Cardiovasc Res. 1993;27:792-800.

23. Damiano RJ Jr, LaFollette P Jr, Cox JL, Lowe JE, Santamore WP.
Significant left ventricular contribution to right ventricular systolic function. Am J Physiol. 1991;261:H1514-24.

24. Farrar DJ, Chow E, Brown CD. Isolated systolic and diastolic ventricular interactions in pacing-induced dilated cardiomyopathy and effects of volume loading and pericardium. Circulation. 1995;92: 1284-90.

25. Pearlman AS, Clark CE, Henry WL, Morganroth J, Itscoitz SB, Epstein SF. Determinants of ventricular septal motion. Influence of relative right and left ventricular size. Circulation. 1976;54:83-91.

26. Kingma I, Tyberg JV, Smith ER. Effects of diastolic transseptal pressure gradient on ventricular septal position and motion. Circulation. 1983;68:1304-14

27. Weyman AE, Wann S, Feigenbaum H, Dillon JC. Mechanism of abnormal septal motion in patients with right ventricular volume overload. A cross-sectional echocardiographic study. Circulation. 1976; 54:179-86.

28. Slinker BK, Glantz SA. End-systolic and end-diastolic ventricular interaction. Am J Physiol. 1986;251:H1062-75.

29. Janicki JS, Weber KT. The pericardium and ventricular interaction, distensibility and function. Am J Physiol. 1980;238:H494-503.

30. Calvin JE. Optimal right ventricular filling pressures and the role of pericardial constraint in right ventricular infarction in dogs. Circulation. 1991;84:852-61.

31. Tyson GS Jr, Maier GW, Olsen CO, Davis JW, Rankin JS. Pericardial influences on ventricular filling in the conscious dog: an analysis based on pericardial pressure. Circ Res. 1984;54:173-84.

\section{Targeted}

The Journal of Thoracic and Cardiovascular Surgery gives you two tables of contents.

The condensed table of contents tells you at a glance what topics and authors are presented each month. The expanded table of contents gives you a brief abstract of each article. You select only those articles of most interest to you for further reading. 